\title{
Strongly Cooperative Nano-CoO/Co Active Phase in Hierarchically Porous Nitrogen-Doped Carbon Microspheres for Efficient Bifunctional Oxygen Electrocatalysis
}

Yajun Ding,c, ${ }^{\mathrm{b}}$, Weiyi Yang, ${ }^{\mathrm{a},}$, Shuang Gao ${ }^{\mathrm{a}}$, Wuzhu Sun ${ }^{\mathrm{d}}$, Caixia Sun ${ }^{\mathrm{e}}$, and Qi Li ${ }^{\mathrm{a}, \mathrm{b} *}$

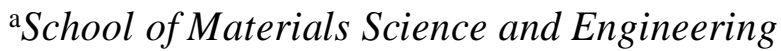

Southwest Jiaotong University, Chengdu 610031, P. R. China

bShenyang National Laboratory for Materials Science

Institute of Metal Research, Chinese Academy of Sciences, Shenyang 110016, P. R. China

'School of Materials Science and Engineering

University of Science and Technology of China, Shenyang 110016, P. R. China

${ }^{\mathrm{d} S c h o o l ~ o f ~ M a t e r i a l s ~ S c i e n c e ~ a n d ~ E n g i n e e r i n g ~}$

Shandong University of Technology, Zibo 255000, P. R. China

${ }^{\mathrm{e}}$ Key Laboratory of New Metallic Functional Materials and Advanced Surface Engineering in

Universities of Shandong and School of Mechanical and Electronic Engineering

Qingdao Binhai University, Qingdao 266555, P. R. China

†These authors contributed equally to this work.

*Corresponding author.

E-mail address: qiliuiuc@outlook.com (Q. Li), Phone: +86-28-87600723.

Postal address: No. 111 Section 1, Northern Second Ring Road, Chengdu, Sichuan Province 610031, P. R. China 


\section{Crystal Structure of Urchin-like Hollow $\mathrm{Co}_{3} \mathrm{O}_{4}$ Microspheres}

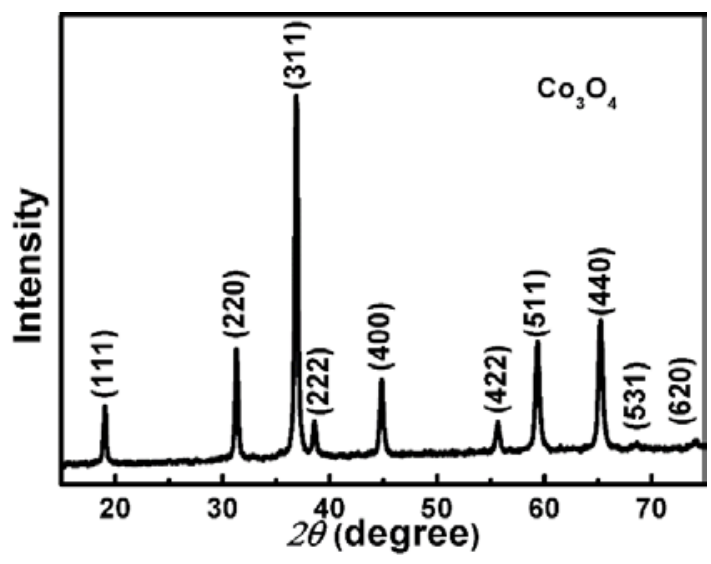

Figure S1. XRD pattern of urchin-like hollow $\mathrm{Co}_{3} \mathrm{O}_{4}$ microspheres.

\section{Chemical Composition of the CoO/Co@N-C Electrocatalyst}

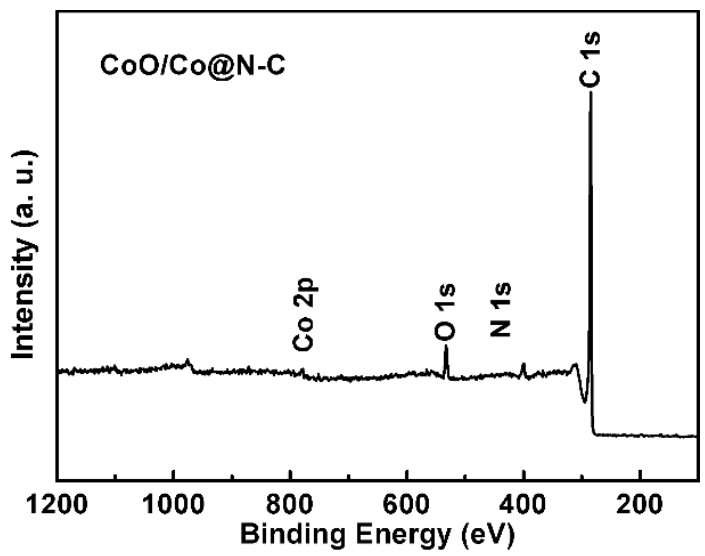

Figure S2. The representative XPS survey spectrum of the CoO/Co@N-C electrocatalyst.

\section{Creation of CoO@N-C and HCl-etched CoO/Co@N-C samples}

The as-obtained CoO/Co@N-C sample was moderately oxidized at $200{ }^{\circ} \mathrm{C}$ for $3 \mathrm{~h}$ under air to create CoO@N-C sample. The as-prepared CoO/Co@N-C sample was etched in a 2 M HCl solution at room temperature for $5 \mathrm{~min}, 20 \mathrm{~min}, 120 \mathrm{~min}$ and $600 \mathrm{~min}$ to create the CoO/Co@N-C-5,CoO/Co@N-C-20,CoO/Co@N-C-120 and CoO/Co@N-C-600 samples, 
respectively. Figure S3 shows SEM image of the CoO@N-C sample, which had very similar morphology to that of CoO/Co@N-C sample. Figure S4 shows their XRD diffraction patterns, compared with that of the as-prepared CoO/Co@N-C sample. It demonstrated clearly that metallic Co was moderately oxidized to $\mathrm{CoO}$ and no diffraction peaks belonged to $\mathrm{CoO}$ could be observed after being etched for 5 min, while the intensities of diffraction peaks belonged to Co became weaker and weaker with the increase of the etching time. Thus, the CoO component in the CoO/Co@N-C sample was removed by etching for just 5 min, while more and more Co was removed with the elongation of the etching time. This observation was in accordance with the ICP-MS measurement results, which showed that the Co content in these samples was 3.82\%, 3.01\%, 0.88\%, 0.06\% and 0.01\% for the etching time of 0, $5 \mathrm{~min}, 20 \mathrm{~min}, 120 \mathrm{~min}$ and 600 min, respectively. Thus, all or almost all of Co was removed from the CoO/Co@N-C sample after being etched for $600 \mathrm{~min}$.

Figure S5 shows the representative elemental mapping images of the CoO/Co@N-C-20 sample obtained from the energy dispersive X-ray spectroscopy (EDS) study on an area with both the dark nanoparticle and lighter color porous matrix. Co signals only existed on the dark nanoparticle while no obvious $\mathrm{O}$ signals could be observed on this dark nanoparticle, which was quite different with that of the CoO/Co@N-C electrocatalyst (see Figure 2c). It suggested that CoO co-existed originally with Co on the dark nanoparticle in the CoO/Co@N-C sample was removed by the etching process. Figure S6 shows the HRTEM observation of the CoO/Co@N-C-20 sample on the interface between Co and N-doped carbon matrix. It demonstrated that the out layer with a light color had an inter-planar spacing of $0.34 \mathrm{~nm}$ corresponding to the (002) plane of graphitic carbon, and the dark nanoparticle had an inter-planar spacing of $0.21 \mathrm{~nm}$ corresponding to the (111) plane of metallic Co. No CoO phase 
could be observed by the HRTEM observation, which further verified that CoO co-existed originally with $\mathrm{Co}$ on the dark nanoparticle in the CoO/Co@N-C sample was removed by the etching process.

During the etching process, these samples kept their hierarchically porous structures. Figure S7 shows a representative SEM image of the CoO/Co@N-C-20 sample, which clearly demonstrated that it was composed of microspheres with abundant macropores penetrated deeply into their inside as the $\mathrm{CoO} / \mathrm{Co} @ \mathrm{~N}-\mathrm{C}$ electrocatalyst. Table S1 summaries the BET specific surface areas, cumulative pore volumes and average pore sizes of these CoO@N-C and HCl-etched samples, compared with that of the as-prepared CoO/Co@N-C sample. They had BET specific surface areas at the similar level, and their cumulative pore volume and average pore size increased only moderately. This observation may be attributed to the connection of mesopores to create macropores during the etching process. Thus, these samples had similar hierarchically porous structures and could be utilized to examine the roles of different components in the $\mathrm{CoO} / \mathrm{Co} @ \mathrm{~N}-\mathrm{C}$ electrocatalyst for the demonstrated efficient ORR performance.

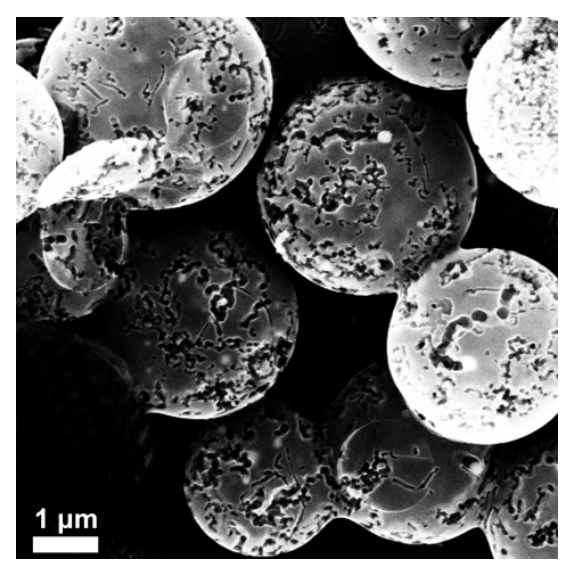

Figure S3. SEM observation of the CoO@N-C sample. 


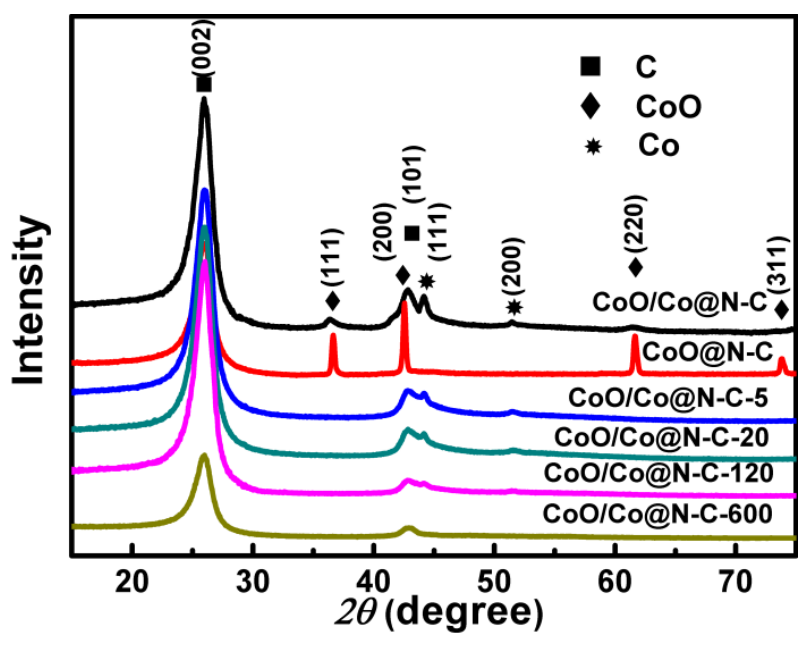

Figure S4. The XRD diffraction patterns of CoO@N-C and HCl-etched CoO/Co@N-C samples with a series of etching time, compared with that of the as-prepared CoO/Co@N-C sample.
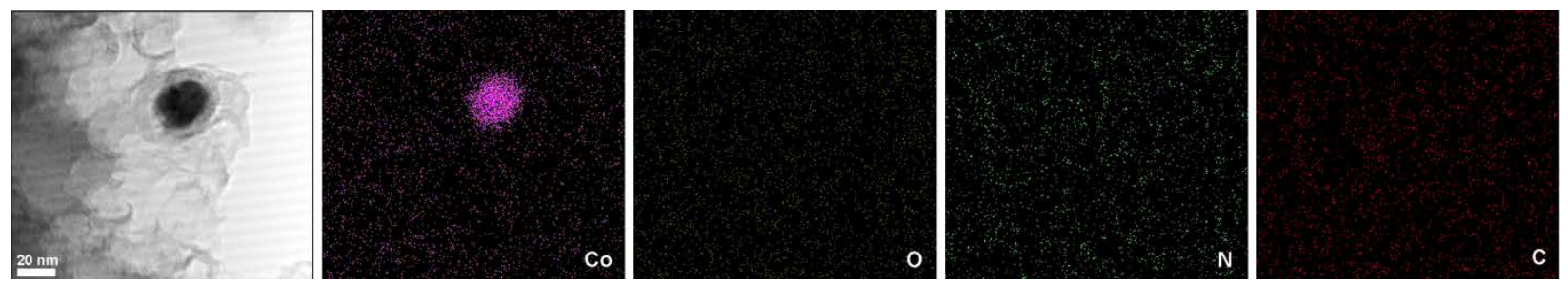

Figure S5. The representative elemental mapping images of the CoO/Co@N-C-20 sample.

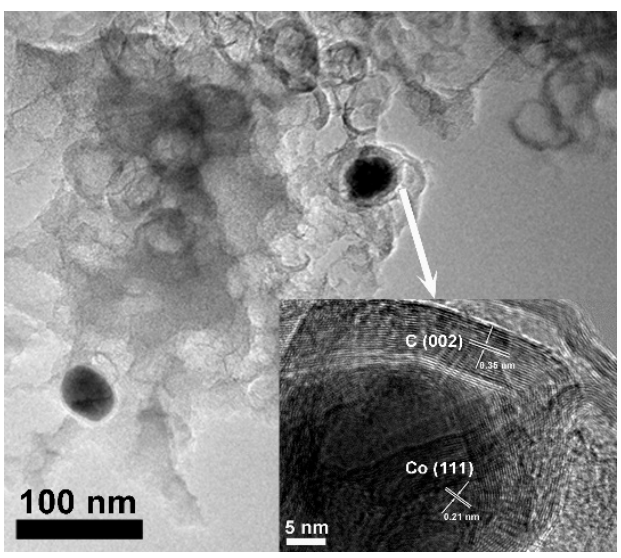

Figure S6. HRTEM observation of the CoO/Co@N-C-20 sample. 


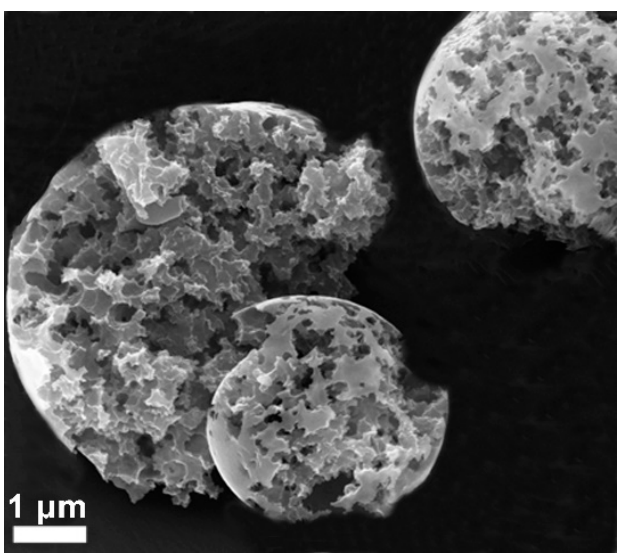

Figure S7. SEM observation of the CoO/Co@N-C-20 sample.

Table S1. The BET specific surface areas, cumulative pore volumes and average pore sizes of these CoO@N-C and HCl-etched samples, compared with that of the as-prepared CoO/Co@N-C sample.

\begin{tabular}{lccc}
\hline Samples & $\begin{array}{c}\text { BET Specific } \\
\text { Surface Area }\left(\mathrm{m}^{2} / \mathrm{g}\right)\end{array}$ & $\begin{array}{c}\text { Specific Pore } \\
\text { Volume }\left(\mathrm{cm}^{3} / \mathrm{g}\right)\end{array}$ & $\begin{array}{c}\text { Average Pore } \\
\text { Size }(\mathrm{nm})\end{array}$ \\
\hline CoO/Co@N-C & 296.58 & 0.44 & 5.97 \\
CoO@N-C & 289.87 & 0.41 & 5.85 \\
CoO/Co@N-C-5 & 312.07 & 0.49 & 6.33 \\
CoO/Co@N-C-20 & 310.11 & 0.51 & 6.57 \\
CoO/Co@N-C-120 & 286.25 & 0.56 & 7.92 \\
CoO/Co@N-C-600 & 275.32 & 0.69 & 8.77 \\
\hline
\end{tabular}

